\title{
ARTIGOS
}

\section{A TOMOGRAFIA COMPUTADORIZADA NA AVALIAÇÃO DO COMPROMETIMENTO ABDOMINAL NA PARACOCCIDIOIDOMICOSE}

\author{
Roberto Martinez, Angela Delete Bellucci e \\ Adhemar Mario Fiorillo
}

\begin{abstract}
A tomografia computadorizada foi utilizada para avaliar o comprometimento abdominal em 25 doentes de paracoccidioidomicose. Existiam lesões intra-abdominais em, respectivamente, $75 \%$ e $23 \%$ dos doentes com as formas aguda ("juvenil") e crônica ("do adulto"). A alteração mais freqüente foi o aumento dos gânglios linfáticos abdominais (12/25 casos); outras lesões foram: calcificação de gânglios linfáticos em 4 casos; obstrução das vias biliares em 5 doentes ictêricos; abscessos ou calcificação esplênica em 3 casos; 2 doentes mostraram lesões incomuns: aumento e irrgularidade do pâncreas em um e múltiplos abscessos nos músculos psoas em outro. Conclui-se que a tomografia computadorizada é procedimento útil na avaliação da disseminaf̧ão da paracoccidioidomicose ao abdome e no diagnóstico de suas complicações abdominais.

Palavras-chaves: Paracoccidioidomicose. Tomografia computadorizada. Linfadenopatia. Vias biliares. Patologia esplênica.
\end{abstract}

Não é infreqüente, nas formas disseminadas da paracoccidioidomicose, a extensão da doença às vísceras abdominais, 1616 existindo também relatos de comprometimento primário com esta localização. ${ }^{2}$ Entre as manifestações clínicas decorrentes deste tipo de lesão incluem-se tumoração intra-abdominal, diarréia, constipação intestinal, icterícia, ascite e abdome agudo ${ }^{7}$. Porém, as lesões de menor tamanho ou localizadas podem ser assintomáticas e mais dificilmente diagnosticadas mesmo ao exame clínico minucioso, tornando subestimada a frequêencia de envolvimento abdominal ${ }^{15}$.

Desde a sua introdução, há poucos anos, a tomografia computadorizada vem se constituindo em método sensível e não invasivo de identificação de anomalias internas. $\mathrm{Na}$ expectativa de que este exame radiológico pudesse avaliar melhor a presença e os tipos de lesões intra-abdominais, ele foi aplicado a uma série de doentes de paracoccidioidomicose, analisando-se os resultados de acordo com a forma clínica da moléstia.

\section{CASUÍSTICA E MÉTODOS}

A tomografia computadorizada foi realizada em 25 pacientes com paracoccidioidomicose ativa, sem tratamento prévio ou apresentando reativação da moléstia, que foram atendidos no Hospital das Clínicas de Ribeirão Preto - SP. O estudo se estendeu durante periodo de 2 anos, constituindo uma série consecutiva de pacientes, independentemente da sintoma-

Trabalho realizado no Hospital das Clínicas da Faculdade de Medicina de Ribeirão Preto - USP -

Departamento de Clínica Médica - 14049 - Ribeirão

Preto - SP.

Recebido para publicação em 12/5/88 tologia abdominal dos mesmos. A serie foi interrompida algumas vezes por problemas técnicos no equipamento.

Os pacientes tinham idade entre 21 e 58 anos, sendo 23 do sexo masculino e 2 do feminino; a cor era branca em 17, preta em 6 e mulata em 2. Para comparação dos resultados eles foram classificados em duas formas clínicas, aguda ou subaguda ("juvenil") e crônica ("do adulto"), de acordo com os critérios adotados no Colóquio Internacional sobre Paracoccidioidomicose, realizado em 1986, na Colômbia ${ }^{10}$. Os 12 pacientes com a forma aguda tinham idade média de 32 anos e apresentavam as seguintes lesões, evidenciadas por exame clínico, radiológico ou endoscópico: ganglionar em 12; cutânea em 4; intestinal em 4; mucosa oral em 1;5 estavam ictéricos. Os 13 doentes com a forma crônica tinham idade média de 47 anos e apresentavam as seguintes localizações: pulmonar em 12; mucosa oral e de vias respiratórias em 9; cutânea em 2; ganglionar em 2; intestinal em 2.

O exame foi realizado com equipamento Delta Scan 2010 da Ohio Nuclear. Com o doente em jejum alimentar, foram realizados cortes tomográficos axiais do abdome após ingestão oral de contraste $(600 \mathrm{ml}$ de solução iodada a $2 \%$ ). Os cortes foram repetidos depois da injeção endovenosa de $100 \mathrm{ml}$ de contraste iodado e da ingestão de mais $200 \mathrm{ml}$ de solução iodada.

\section{RESULTADOS}

A tomografia computadorizada detectou anomalias em 12 (48\%) dos doentes (Tabela 1). Elas foram mais freqüentes na forma aguda (75\% dos doentes), mas também ocorreram nos casos de forma crônica $(23 \%)$.

A Tabela 2 relaciona os tipos de lesões encontradas nos 12 doentes com anomalias na tomografia 
Martinez $R$, Bellucci $A D$, Fiorillo AM. A tomografía computadorizada na avaliação do comprometimento abdominal na paracoccidiodomicose. Revista da Sociedade Brasileira de Medicina Tropical 21, 47-50, Abr-Jun, 1988.

Tabela 1 - Freqüencia, de acordo com a forma clínica, de doentes de paracoccidioidomicose com anomalias abdominais detectadas pela tomografia computadorizada (TC).

\begin{tabular}{lccc}
\hline Forma de doença & $\begin{array}{c}\text { N. de } \\
\text { doentes }\end{array}$ & $\begin{array}{c}\text { TC normal } \\
n^{\circ}(\%)\end{array}$ & $\begin{array}{l}\text { TC alterado } \\
n^{\circ}(\%)\end{array}$ \\
\hline $\begin{array}{l}\text { Aguda ("juvenil") } \\
\text { Crônica ("do adulto") }\end{array}$ & 12 & $\begin{array}{r}3(25,0) \\
10(76,9)\end{array}$ & $\begin{array}{l}9(75,0) \\
3(23,1)\end{array}$ \\
\hline Todos os pacientes & 25 & $13(52,0)$ & $12(48,0)$ \\
\hline
\end{tabular}

Tabela 2 - Tipos de alterações reveladas pela tomografia abdominal em 25 doentes de paracoccidioidomicose.

\section{Alteração Radiológica}

N. de

Doentes

$\%$

1) Aumento de gânglios linfáticos na região peri-aórtica no mesentério no hilo hepático

2) Calcificação de gânglios linfáticos 4 16

3) Dilatação das vias biliares intra e extra-hepáticas intra-hepáticas

4) Alterações no baço

abscessos

calcificação

5) Aumento e irregularidade da cabeça do pâncreas

6) Abscessos nos músculos psoas havia aumento ou calcificação de gânglios linfáticos do hilo hepático (Figura 2) e em 3 pôde ser localizado no colédoco terminal o local de obstrução das vias biliares. Nāo foram encontradas lesões do parênquima hepático nestes 5 e nos casos restantes.

Em 2 doentes havia formações hipodensas no baço compativeis com múltiplos microabscessos em um caso e com vários abscessos de maior tamanho, com diâmetro máximo de $50 \mathrm{~mm}$, em outro (Figura 3 ). Um terceiro doente mostrou áreas de calcificação esplênica. Entre as lesões menos freqüentes, aumento e irregularidade da cabeça do pâncreas foram notados em um doente, que também apresentava adenopatia abdominal e dilatação das vias biliares; outro doente tinha vários abscessos em ambos os músculos psoas, o maior deles com $76 \mathrm{~mm}$ de diâmetro, juntamente com linfadenopatia abdominal (Figura 4). Quanto aos rins, existiam cálculos pequenos em 2 pacientes e formações císticas em 2 outros.

Analisando os tipos de lesões de acordo com a classificação clínica da paracoccidioidomicose, nos 3 doentes com a forma crônica e tomografia anômala havia apenas adenomegalia periaórtica, associada em

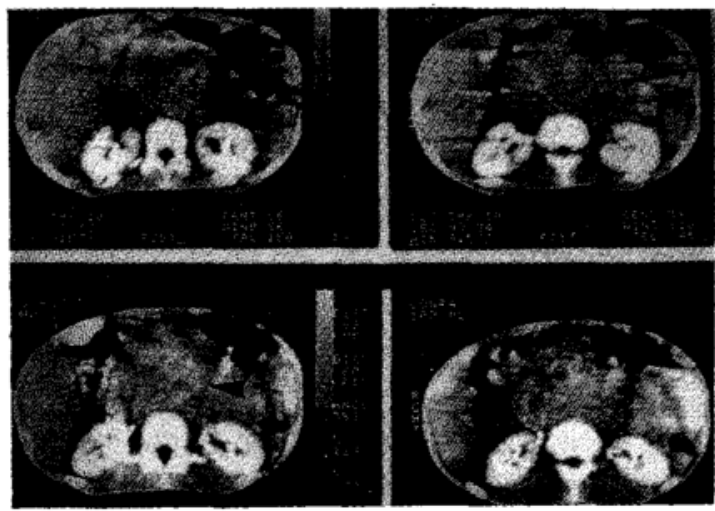

Figura 1-Extensa adenomegalia periaórtica e pericaval.

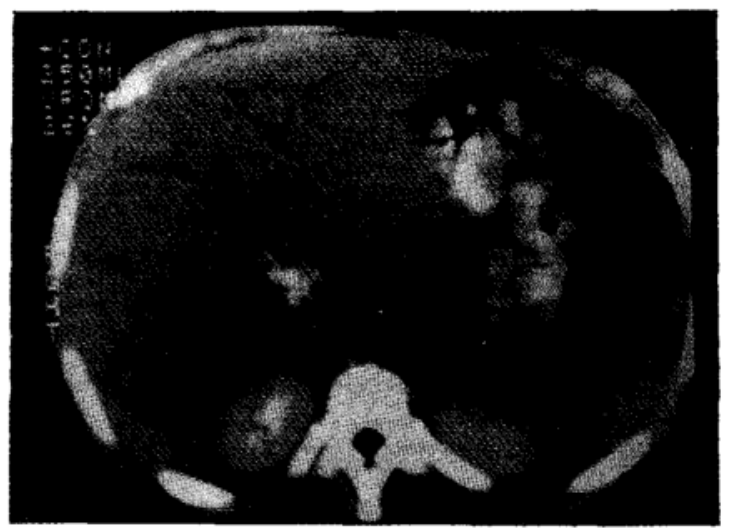

Figura 2-Dilatação das vias biliares e adenomegalia $e$ calcifícação na região do hilo hepático. computadorizada. Em todos eles havia irregularidades nos gânglios linfáticos abdominais, que se encontravam aumentados, chegando a constituir massas volumosas, em 11 casos ( 3 também com calcificações); no caso restante, havia apenas calcificação difusa dos linfonodos. As irregularidades situavam-se principalmente na cadeia ganglionar peri-aórtica (Figura 1).

Os 5 pacientes com icterícia tinham dilatação das vias biliares intra-hepáticas, em 3 dos quais tambem das vias extra-hepáticas. Em 4 destes 5 pacientes 


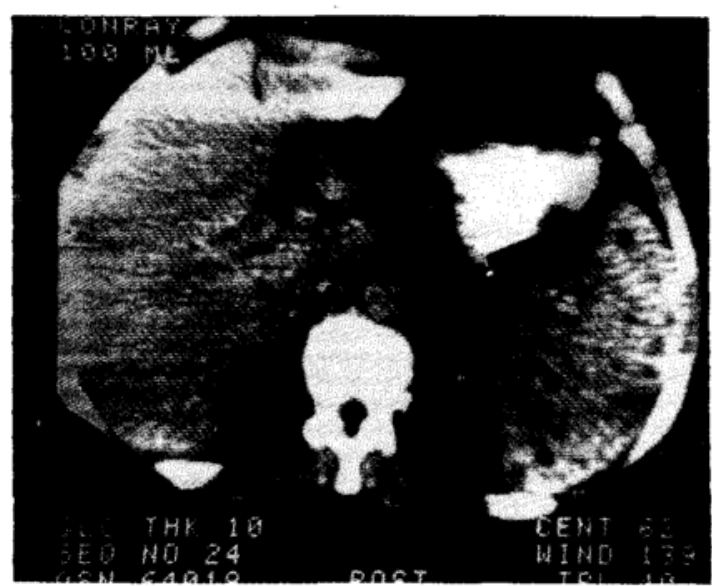

Figura 3-Múltiplos abscessos no baço.

um deles com calcificação ganglionar. As outras alterações foram todas observadas em casos de forma aguda.

\section{DISCUSSÃO}

A utilização da tomografia computadorizada nesta série de 25 doentes de paracoccidioidomicose permitiu evidenciar comprometimento intra-abdominal em praticamente metade dos casos. Em parte deles já havia clinicamente este diagnóstico, inferido pela presença de massa palpável no abdome ou pela ictericia. $\mathrm{O}$ achado tomográfico surpreendeu, porém, em 3 doentes pela inexistência de dados clínicos ou de exames complementares que sugerissem a disseminação da moléstia, a qual se localizava aparentemente apenas nas vias respiratórias ou em cadeias linfáticas superficiais. Em um destes casos, a única lesão aparente era uma ulceração na mucosa buco-faringeana, sendo descoberta uma adenomegalia periaórtica. A capacidade de certos exames especializados em revelar e detalhar novas lesões já foi demonstrada anteriormente na paracoccidioidomicose. Assim, a própria tomografia computadorizada foi empregada na detecção de lesões do sistema nervoso central ${ }^{14}$, do mesmo modo que a ultrassonografia em relação ao abdome ${ }^{5}$ ou a endoscopia digestiva no diagnóstico de lesões intestinais assintomáticas ${ }^{13}$. Estes procedimentos, quando aplicados a séries de casos consecutivos, não selecionados pela sintomatologia, podem trazer esclarecimentos importantes sobre a patogenia da moléstia, ao mostrarem as lesões iniciais conseqüentes à disseminação fúngica.

As anomalias verificadas foram mais comuns na forma aguda da paracoccidioidomicose, o que está de acordo com os dados clínicos, uma vez que porção significativa destes pacientes mostra sinais de lesão intra-abdominal, a qual pode até representar manifestação isolada da moléstia - forma linfático-abdomi-

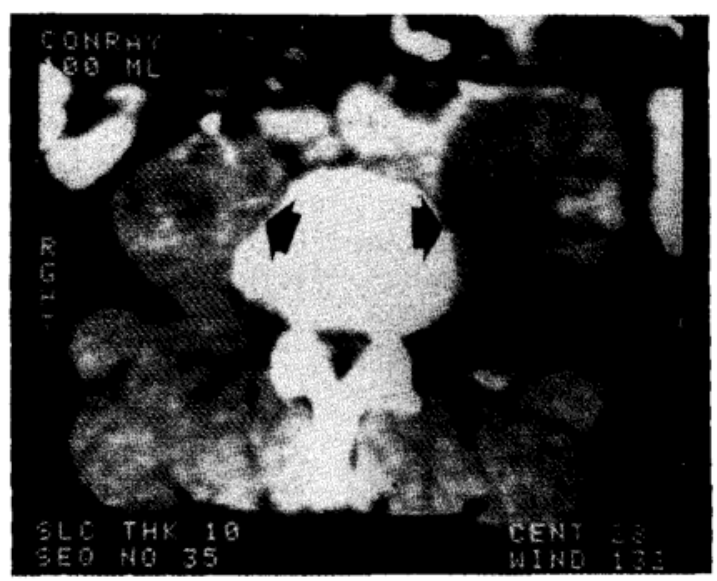

Figura 4-Grandes abscessos no músculo psoas à esquerda $e$ abscessos menores à direita.

nal $^{2}$. A disseminação preferencial do Paracoccidioides brasilienses por via linfática ${ }^{15}$ pode explicar a alta freqüência de adenopatia observada na forma aguda e também de lesões em outras vísceras, que se supõe possam resultar secundariamente da lesão ganglionar $^{712}$.

Os cortes tomográficos permitiram esclarecer a causa da ictericia em 5 doentes, que foi considerada do tipo obstrutivo pela dilatação das vias biliares e pela presença de gânglios linfáticos alterados no hilo hepático, juntamente com ausência de lesões no parênquima hepático. A compressão de vias biliares por gânglios regionais enfartados ou o seu envolvimento direto no processo granulomatoso são as causas mais comuns de ictericia na paracoccidioidomicose huma$\mathrm{na}^{311}$.

Apesar da esplenomegalia ser percebida em parte dos pacientes, clinicamente é dificil ter certeza de lesão direta do baço pelo $P$. brasiliensis. A descoberta de 2 pacientes com abscessos e outro com calcificações no órgão ( $12 \%$ dos casos) mostra o potencial da tomografia para evidenciar tais lesões. Em duas séries de casos necropsiados no Estado de São Paulo, o percentual de comprometimento esplênico foi de $39,3 \%^{6}$ e de $29,0 \%{ }^{9}$.

Os achados inesperạdos da investigação foram a lesão pancreática em um doente com icterícia e os abscessos nos músculos psoas em outro caso. A lesão do pâncreas é rara e pode se apresentar com características que lembram um carcinoma pancreático, trazendo dificuldades para o diagróstico, mesmo com a realização de laparotomia exploradora ${ }^{4} 8.0$ paciente com abscessos nos psoas, apesar de estar em tratamento anti-fúngico já há alguns meses, mantinha febre e adenomegalia periférica, sem contudo apresentar sintomatologia que permitisse diagnosticar a lesão muscular.

Os resultades deste estudo levam à conclusão de que o comprometimento intra-abdominal na paracoc- 
Martinez $R$, Bellucci AD, Fiorillo AM. A tomografía computadorizada na avaliação do comprometimento abdominal na paracoccidiodomicose. Revista da Sociedade Brasileira de Medicina Tropical 21, 47-50, Abr-Jun, 1988.

cidioidomicose é mais freqüente do que indicam as manifestações clínicas, sugerindo também que a propagação do fungo e as lesões iniciais no abdome ocorram no sistema linfático. A tomografia é um método sensivel na deteç̧ão de adenomegalia ou de outras lesões abdominais extra-intestinais, sendo bastante útil no esclarecimento dos casos de icterícia, de complicações abdominais e de resposta insuficiente à terapêutica.

\section{SUMMARY}

Computerised tomography was utilized in 25 paracoccidioidomycosis patients to evaluate abdominal involvement. This was observed in patients, $75 \%$ of acute (juvenile type) and 23\% of chronic (adult type) patients. The most frequent alteration was abdominal lymphnode enlargement (12/25 cases). Other lesions consisted of lymphnode calcification in 4 cases, biliary tract obstruction in 5 icteric patients, splenic abscess or calcification in 3 cases. Two patients had unusual lesions: one with pancreatic enlargement and irregularity and other with multiple abscesses in both psoas muscles. It was concluded that computerised tomography is a useful procedure for evaluating paracoccidioidomycosis dissemination to the abdomen and for the diagnosis of its abdominal complications.

Key-words: Paracoccidiodomycosis. X-ray computerised tomography. Limphatic disease. Biliary tract and spleen pathology.

\section{REFERÊNCIAS BIBLIOGRÁFICAS}

1. Andrade DR, Hutzler RV, Carvalho SA, Rosenthal C, Carvalho MAB, Ferreira JM. Hipoproteinemia em pacientes com paracoccidioidomicose do tubo digestivo e sistema linfático abdominal: revisāo de casos de necropsia e apresentação de um caso com perda protéica digestiva. Revista do Hospital das Clínicas da Faculdade de Medicina da Universidade de São Paulo 31:174-179, 1976.

2. Barbosa W, Daher R, Oliveira AR. Forma linfáticoabdominal da blastomicose sul-americana. Revista do Instituto de Medicina Tropical de São Paulo 10:16-27, 1968.

3. Boccalandro I, Mello e Albuquerque FJ. Ictericia e comprometimento hepático na blastomicose sul-americana. A propósito de 10 casos. Revista Paulista de Medicina 56:350-366, 1960.
4. Castro RM, Del Negro G, Bassoi ON, Faria CV. Dificuldades diagnósticas na blastomicose sul-americana. A propósito de oito casos. Revista Paulista de Medicina 53:479-496, 1958.

5. Cerri GG, Del Negro G, Magalhães AJR, Amato Neto V, Magalhães A. Utilização da ultrassonografia e da linfografia na forma linfática da paracoccidioidomicose. Revista do Hospital das Clínicas da Faculdade de Medicina da Universidade de São Paulo 38:160-163, 1983.

6. Del Negro G. Paracoccidioidomicose (blastomicose sulamericana). Aspectos clinicos. Ars Curandi 7:30-36, 1975.

7. Fiorillo AM, Martinez R, Moraes CR. Lesões do aparelho digestivo. In: Del Negro G, Lacaz CS, Fiorillo AM. (ed) Paracoccidioidomicose (blastomicose sulamericana). Sarvier-Edusp, São Paulo, p. 179-193, 1982.

8. Forattini OP. Blastomicose da região pancreática. Revista Paulista de Medicina 31:165-172, 1947.

9. Franco MF, Montenegro MRG. Anatomia patológica. In: Del Negro G, Lacaz CS, Fiorillo AM. (ed) Paracoccidioidomicose (blastomicose sul-americana). Sarvier-Edusp, São Paulo, p. 97-117, 1982.

10. Franco M, Montenegro MR, Mendes RP, Marques SA, Dillon NL, Mota NGS. Paracoccidioidomycosis: a recently proposed classification of its clinical forms. Revista da Sociedade Brasileira de Medicina Tropical 20:129-132, 1987.

11. Goffi FS, Ferrarini E, São Thiago JB, Silva LC. Ictericia obstrutiva por granuloma blastomicótico do colédoco. Revista Paulista de Medicina 57:12-21, 1960.

12. Martinez R, Meneghelli UG, Dantas RO, Fiorillo AM. $O$ comprometimento gastrintestinal na blastomicose sul-americana (paracoccidiodomicose). I. Estudo clínico, radiológico e histopatológico. Revista da Associação Médica Brasileira 25:31-34, 1979.

13. Martinez R, Módena JLP, Barbieri-Neto J, Fiorillo AM. Avaliação endoscópica do comprometimento de esôfago, estômago e duodeno na paracoccidioidomicose humana. Arquivos de Gastroenterologia de São Paulo 23:21-25, 1986.

14. Minguetti G. Tomografia computadorizada dos granulomas blastomicóticos encefálicos. Revista do Instituto de Medicina Tropical de São Paulo 25:99-107, 1983.

15. Padilha Gonçalves A. Localizações ganglionares da micose de Lutz (Blastomicose brasileira). Boletim da Academia Nacional de Medicina 134:5-17, 1962.

16. Teixeira F, Gayotto LC, Britto T. Morphological patterns of the liver in South American blastomycosis. Histopathology 2:231-237, 1978. 